\title{
Psicología en Chile: análisis de su historia, presente y futuro*
}

\section{Psychology in Chile: Analysis of its History, Present and Future}

Recibido: 14 de agosto de 2014 | Revisado: 10 de febrero de 2015 | Aceptado: 10 de julio de 2015

\author{
AlFONSO URZÚA ** \\ Universidad Católica del Norte, Antofagasta, Chile \\ Pablo Vera-Villarroel *** \\ Universidad de Santiago de Chile, Santiago, Chile \\ Claudia ZÚÑIGA ***** \\ Universidad de Chile, Santiago, Chile \\ GONZALO SALAS ****** \\ Universidad Católica del Maule, Talca, Chile
}

doi:10.11144/Javeriana.upsy14-3.pcah

Para citar este artículo: Urzúa, A., Vera-Villarroel, P., Zúñiga, C., \& Salas, G. (2015). Psicología en Chile: análisis de su historia, presente y futuro. Universitas Psychologica, 14(3), 1125-1142. http:// dx.doi.org/10.11144/Javeriana.upsy14-3.pcah

Artículo de reflexión

** Académico Escuela de Psicología, Universidad Católica del Norte. Avenida Angamos 0610, Antofagasta, Chile. Correo electrónico: alurzua@ ucn.cl

**** Académico Escuela de Psicología, Universidad de Santiago de Chile. Avda. Ecuador 3650, tercer piso. Estación Central, Santiago, Chile. Correo electrónico: pablo.vera@usach.cl

****** Académica Departamento de Psicología, Universidad de Chile. Av.Capitán Ignacio Carrera Pinto 1045, Nuñoa, Santiago, Chile. Correo electrónico: cczuniga@u.uchile.cl

****** Académico Departamento de Psicología, Universidad Católica del Maule. Avda. San Miguel 3605, Talca.Correo electrónico: gsalas@ucm.cl

\section{RES UMEN}

El presente artículo realiza una revisión del desarrollo y estado actual de la psicología chilena. En 1946 se instaura formalmente la formación de psicólogos en Chile; en 1959 se oficializa la Asociación de Psicólogos de Chile y en 1968 se funda el Colegio de Psicólogos de Chile. Esto sitúa a Chile dentro de los primeros países de la región en el desarrollo formal de la disciplina. Actualmente, existen 139 programas de formación en psicología, impartidos en 49 universidades; sumado a una creciente oferta de programas de magíster y doctorado en psicología. Con respecto al desarrollo científico de la disciplina, se aprecia un alza sostenida en el número de artículos publicados, destacando que desde el año 2011 se sobrepasan los 100 artículos por año, aunque esta producción está ligada a unas pocas instituciones y autores con alto protagonismo. Adicionalmente, se observa una baja colaboración entre investigadores chilenos y latinoamericanos en estudios y productividad científica, a pesar de compartir temáticas y problemas similares. Respecto a los desafíos futuros, es necesario aumentar los sistemas de aseguramiento de la calidad en pre y posgrados, así como la mayor influencia de los psicólogos y psicólogas en la sociedad.

Palabras clave

psicología; desarrollo de la psicología; historia de la psicología

\section{A B S T R ACT}

This paper present a review of the development and current state of Chilean psychology. In 1946 formally establishes the formation of psychologists in Chile; in 1959, it made official the Association of Psychologists of Chile and in 1968 the College of Psychologists of Chile was founded. This puts Chile in the first countries in the region in the formal development of the discipline. Currently, there are 139 training programs in psychology, taught in 49 universities. Added to that, a growing supply of master's and doctoral programs in psychology. With regard to the scientific development of the discipline shown a sustained increase in the number of published articles, noting that since 2011 has exceeded 100 items per year, although this production is linked to a few institutions and authors with high prominence. Additionally, a low collaboration between Chilean and Latin American researchers in studies and scientific productivity, despite sharing similar issues and problems observed. Regarding future challenges, it is necessary to increase systems quality assurance in pre and postgraduate courses, as well as the most influential psychologists of society.

Keywords

Psychology; Development of Psychology; History of Psychology 


\section{Antecedentes históricos}

\section{Prolegómenos de la psicología científica}

La psicología en Chile ha existido desde los tiempos de la Conquista y la Colonia, ya que los Jesuitas, Franciscanos y Domínicos la desarrollaron con asiduidad en el período hispánico (Hanisch, 1963); sin embargo, su relación con la filosofía se remite a los orígenes de la Universidad de Chile, específicamente a finales del siglo XIX, dado el énfasis científico que atesoraba la ciencia psicológica en el país y en el mundo.

Desde 1843, cuando se funda la Universidad de Chile, Andrés Bello demostró una gran preocupación por el avance de las discusiones psicológicas que se plasmaban del asociacionismo forjado en Londres por James Mill (Instituto de Chile, 1982; Jaksic, 2001) y la escuela escocesa de Thomas Reid (Alarcón, 2002). Por su parte, la enseñanza de la psicología habría comenzado en el año 1852 en las cátedras de Filosofía de la Universidad de Chile, donde se incluyó la enseñanza de la psicología en la formación de los estudiantes de medicina, debido a la vinculación que existía entre psicología y fisiología (Pizarro, 1999). En las postrimerías del siglo XIX, son importantes los trabajos de los profesores Schneider y Villalobos acerca del origen de las sensaciones y psicología aplicada, publicados en 1890 y 1893, respectivamente (Calderón, 2000).

Uno de los ideales decimonónicos que se abrió paso fue el positivismo francés que inició Auguste Comte, el cual tuvo un impacto relevante en América Latina y específicamente en naciones como Brasil, Argentina, México y también Chile (Alarcón, 1997). Esta posición fue considerada principalmente por José Victorino Lastarria, Juan Serapio Lois y Valentín Letelier, entre otros. El trabajo de Letelier, relacionado directamente con lo psicológico fue descrito principalmente en su obra Filosofía de la educación, donde refirió la relevancia de la psicología pedagógica y, citando a Van Biervliet, profesor de psicología experimental de la Universidad de Gante, analizó cuestiones relacionadas con la adaptabilidad individual de la enseñanza, la disciplina de la atención, la posibilidad de evitar las distracciones, la pereza y las condiciones del estudio mnemónico (Letelier, 1927).

Los minerales del norte, en especial Chañarci1lo, con el enorme impulso económico que dieron a la República apenas treinta años después de su fundación, determinaron la inmigración alemana (Venturino, 1929), y la psicología no fue la excepción, ya que varios de estos inmigrantes hicieron importantes aportes a ella. Primero, Elisabeth Isabel Bongard en la Escuela Normal de La Serena (Alfonso \& Pacheco, 2011) y, posteriormente, Georg Heinrich Schneider y Wilhelm Mann con la creación del Instituto Pedagógico de la Universidad de Chile en el año 1889 (Salas \& Lizama, 2013). Es así como se observa en esa época una lógica de la psicología aplicada al campo educativo, donde el conocimiento psicológico fue relevante para generar un auxilio a la educación (Parra, 2015; Salas \& Inzunza, 2013), de esto se desprende que la psicología educacional fue la primera de las áreas de desarrollo de la psicología en el país.

En 1905, se creó en la Escuela Normal de Copiapó el primer laboratorio de psicología experimental en Chile (Miguez, Betancourt, \& Vera-Villarroel, 2010), mediante el arduo trabajo de Rómulo Peña Maturana quien intentó medir la inteligencia, el vigor intelectual y la resistencia física (Poblete, 1980, 1995; Salas, 2012). En dicho laboratorio de corte naturalista, se constituyó una "disciplina del espíritu" que seguía principalmente las directrices de Wundt. En 1908 Wilhelm Mann crea el Laboratorio de Psicología de la Universidad de Chile (Salas \& Lizama, 2013) que fue transformado desde 1923 en un gabinete ocupado principalmente en la medición de la inteligencia, mediante la adaptación de la Escala Binet-Simon a la realidad chilena (Tirapegui, 1928).

La investigación aplicada comienza a desarrollarse de forma científica con la aparición del Instituto de Psicología de la Universidad de Chile, en 1941, creado por Abelardo Iturriaga, quien fue formado en Francia por Henry Wallon (Munizaga \& Cizaletti, 1967). Esta entidad tuvo por objeto investigar en los campos de la psicología experimental y diferencial, la psicología aplicada al derecho, a la educación, al trabajo profesional y a la higiene mental (Iturriaga, 1944). 
Solo con unos años de anterioridad, en 1938, Juan Marín visualizaba al psicoanálisis conectado con la higiene mental, lo cual permitía imaginar una humanidad sin locos ni neuróticos (Ruperthuz, 2012). En esta misma línea, aparecen trabajos sobre higiene mental, su relación con la psiquiatría (Cubillos \& Agüero, 1941) y la necesidad de una legislación sobre asistencia a enfermos mentales que mejorara tanto el trato a los pacientes como el contexto de las prácticas (Horvitz, 1946).

\section{La psicología y la formación de psicólogos}

El Decreto 1023 del 20 de agosto de 1946 instaura la formación de psicólogos en Chile (Descouvieres, 1999). Junto a Colombia, es el primer país del Cono Sur de América en dictar la carrera de psicología, la cual comienza en 1947 con un Curso Especial de Psicología, como se relata en el Informe Nassar (Salas, 2014a). En este informe, se pueden observar los prolegómenos de cuatro especialidades clásicas de la psicología (educacional, clínica, laboral y social-comunitaria), y la inclusión temprana de una práctica profesional y una tesis, como requisitos para la titulación. El plan de estudios inicial fue modificado por primera vez en 1949 (Nassar, 1955). Ya en el año 1952, se tituló la primera generación de psicólogos que incluía a Eugenio Aragón, Teresa Cumsille, Víctor Donaire, Herminio García, Liana Ortiz y Jorge Valenzuela (Ligueño, Parra, Moncada, \& Laborda, 2010).

En los años 50, Américo Foradori escribe un completo reporte sobre la psicología en América y en el apartado sobre Chile, aparecen datos relevantes de lo ocurrido en la ciudad de Concepción con la psicología, que se enseñaba en la Escuela de Educación como materia obligatoria para los estudiantes de la carrera de profesorado de instrucción primaria y secundaria. La enseñanza se adaptaba en líneas generales a los planes propuestos por el Instituto Pedagógico y se incluían cursos de psicología pedagógica, psicología de la adolescencia y mediciones mentales, de carácter obligatorio para los profesores de enseñanza secundaria. Los profesores de enseñanza primaria por su parte, debían aprobar cursos de psicología general, psicología del niño y psicología diferencial (Foradori, 1954).

En la Universidad Católica, se crea el Departamento de Psicología en el año 1954 mediante el liderazgo del psicólogo húngaro Bela Szekely, quien tuvo un fugaz paso por Chile (Bravo, 2004). Al año siguiente, asumió la dirección Eduardo Rosales, quien de inmediato viajó a Europa a estudiar la organización de los principales centros psicológicos de Italia, Bélgica, Francia y España, y además solicitó informes a universidades norteamericanas y a algunos países de Sudamérica (Rosales, marzo 27, 1955). En sus inicios, estos cursos tuvieron una mirada científica y experimental, lo cual fue mutando hacia los enfoques psicodinámicos y fenomenológicos (Luco, 2010). La Universidad de Chile y la Universidad Católica eran, en aquel entonces, modelos de institución de educación superior competitivos, rivales y opuestos (Villegas, 2012).

Pasaron largos años para que la psicología se desarrollara en otras universidades. En el campo de las universidades del Estado, la Universidad de la Frontera abre la carrera en Temuco en el año 1982 y la Universidad de Concepción, una universidad privada, que ostenta una clara vocación pública, lo hace el 1985. Además, desde el año 1981, cuando se promulga la Ley General de Universidades, se generó una amplia explosión de programas de psicología en universidades privadas (Salas, 2014b; Urzúa, 2008).

En cuanto a la asociatividad, en el año 1968 se crea el Colegio de Psicólogos, institución referente que tuvo el carácter aglutinador y nacional de los psicólogos chilenos hasta su eliminación en 1973 , aunque reabre años más tarde su funcionamiento como asociación gremial hasta la actualidad (Urra, 2008). El Colegio tuvo su origen en la Asociación de Psicólogos de Chile, creada en 1952, y fue oficializado en 1959 (Descouvieres, 1985; Kalawski, Sepulveda, \& Célis, 1996). Estas asociaciones fueron las primeras relacionadas con la psicología, ya que solamente la Asociación Psicoanalítica de Chile, creada en 1949 (Salas, 2014b) o la Sociedad Chilena de Sexología Antropológica en 1965 que reunía a psicólogos, médicos y representantes de disciplinas afines, tienen una data más antigua (Ba- 
rrientos, Palma, \& Gómez, 2014). Posteriormente, se han creado diversas sociedades científicas en las diversas áreas de la psicología, las cuales se exponen más adelante.

En la misma época de creación del Colegio, se observa el auge del modelo conductista en Chile, ya que el regreso de Sergio Yulis al país en plena época de la reforma universitaria de 1968, permitió la incorporación de la terapia comportamental en cursos de psicoterapia (Quezada, Vergés, \& Laborda, 2014). Yulis llegó a un ambiente convulsionado por cambios, en el cual los estudiantes de psicología comenzaban a construir un discurso de participación que se reflejó en transformaciones importantes (Salas \& Lizama, 2013). Solo unos años después, con la instauración de la dictadura militar, inevitablemente el desarrollo alcanzado por la psicología se mermó, lo cual se pudo apreciar, solo a modo de ejemplo, en el cierre de los recién creados Departamentos de Psicología Social y el exilio de numerosos profesores. La fuga de conocimiento, los miedos y la autocensura, por parte de muchos de los que permanecieron en el país, estancó el desarrollo profesional e investigativo (Mansur \& Nóbrega, 1999).

De cualquier forma, el primer encuentro de psicólogos se realizó el año 1977 (Morales, Díaz, Scharager \& Szkilai, 1988) y el primer Congreso Nacional de psicólogos se realizó en 1985 (Colegio de Psicólogos, 1985). Desde la fecha solo se han llevado a cabo siete de estos encuentros en los años 1985, 1987, 1989, 1991, 1997, 1999 у 2007, sin embargo, dichos espacios comenzaron a permitir la apertura de la investigación en psicología, ya que en 1978, se creó la hoy desaparecida Revista Chilena de Psicología y en 1982 Terapia Psicológica (revista de la Sociedad Chilena de Psicología Clínica), primera y única revista nacional indexada a la Web of Science hasta hoy. Respecto a los congresos, también se debe destacar que un buen número de ellos se realiza en las diversas especialidades de la disciplina, lo que se suma a la regularidad anual de los congresos de la Sociedad Chilena de Psicología Clínica y de la Red de Escuelas de Psicología del Consorcio de Universidades Estatales, que se lleva a cabo desde el año 2006 (Juliá, 2013), denotan un panorama expansivo y distintivo que permite a los profesionales, inves- tigadores y estudiantes de psicología seleccionar los espacios de acuerdo a sus intereses.

Finalmente, cabe destacar que a partir de la inclusión del psicólogo en la salud pública, a través del Código Sanitario de 1993 (Loubat, 2013), se ha posibilitado el desarrollo del rol del psicólogo en distintos programas vinculados al Ministerio de Salud; y algo similar acontece en educación con la creación del Decreto 170 (Ministerio de Educación, 2009) que, aunque existen legítimas y evidentes críticas a su funcionamiento, permite la contratación masiva de psicólogos educativos en el campo de las necesidades especiales. En el ámbito laboral y social comunitario, falta mucho por desarrollar en políticas de gobierno.

Respecto a la investigación científica en psicología en Chile, se sugiere revisar los dos tomos escritos por Villegas y Rodríguez, donde se entrevista a destacados investigadores de los campos de psicología básica y social (Villegas \& Rodríguez, 2005) y de las áreas de clínica, educacional y organizacional (Rodríguez \& Villegas, 2007), quienes refieren los respectivos avances. También son relevantes los estudios sobre la investigación actual de la psicología clínica en Chile (Vera-Villarroel \& Lillo, 2006) y otro donde se compara la investigación de la psicología clínica nacional con la producción argentina (Vera-Villarroel \& Mustaca, 2006) e iberoamericana (Lillo \& Martini, 2013).

\section{Actualidad}

\section{Formación de pregrado}

De acuerdo a lo informado por el Consejo Nacional de Educación (CNE), dependiente del Ministerio de Educación chileno (CNE, 2015), actualmente, existen 139 programas de formación en psicología, impartidos en 49 universidades. $\mathrm{Al}$ año 2008, se reportaban 87 programas (Urzúa, 2008), lo que implica la creación de 52 programas en los últimos siete años, es decir, un promedio anual de siete programas nuevos. Lo anterior es preocupante no tan solo porque no se asegura la empleabilidad de los posibles graduados, sino también porque no todos los programas de formación cuentan con las 
medidas mínimas de aseguramiento de la calidad, como lo es el someterse al proceso de acreditación, el cual actualmente es obligatorio solo para carreras de medicina y pedagogías.

Según la Comisión Nacional de Acreditación del Gobierno de Chile (CNA, 2015), a junio del 2015 se han presentado a acreditación solo 32 centros formadores de pregrado con acreditaciones que van desde 0 (es decir, no son acreditados) a 7 años (máximo permitido por ley), es decir, solo un $65 \%$ de las instituciones. En la Tabla 1, se puede observar el número de programas por universidad impartiendo psicología en Chile, donde es notoria la presencia de tres universidades con una alta cantidad de programas en distintas sedes $(13,17 \mathrm{y}$ 19 programas cada una de ellas).

No se tiene certeza del número exacto de psicólogos titulados en Chile, sin embargo, se sabe que la cantidad de estudiantes matriculados en programas de psicología ha aumentado en un $48 \%$ en los últimos 10 años, de acuerdo a datos proporcionado por el Consejo Nacional de Educación (CNED, 2015). En el Gráfico 1, se pueden apreciar los matriculados por año.

Tabla 1

Universidades que imparten psicología en Chile, años de acreditación y número de programas de formación que imparte cada una de ellas

\begin{tabular}{|c|c|c|c|c|}
\hline & & Años & Progr. & Tipo de Universidad \\
\hline 1 & Pontificia Universidad Católica de Chile & 7 & 1 & Privada, Consejo de Rectores* \\
\hline 2 & Universidad de Talca & 7 & 1 & Estatal, Consejo de Rectores \\
\hline 3 & Pontificia Universidad Católica de Valparaíso & 6 & 1 & Privada, Consejo de Rectores \\
\hline 4 & Universidad Católica del Norte & 6 & 1 & Privada, Consejo de Rectores \\
\hline 5 & Universidad de Chile & 6 & 1 & Estatal, Consejo de Rectores \\
\hline 6 & Universidad de Concepción & 6 & 1 & Privada, Consejo de Rectores \\
\hline 7 & Universidad de la Frontera & 6 & 1 & Estatal, Consejo de Rectores \\
\hline 8 & Universidad de la Serena & 6 & 1 & Estatal, Consejo de Rectores \\
\hline 9 & Universidad de Santiago de Chile & 6 & 1 & Estatal, Consejo de Rectores \\
\hline 10 & Universidad Diego Portales & 6 & 1 & Privada \\
\hline 11 & Universidad Adolfo Ibáñez & 5 & 2 & Privada \\
\hline 12 & Universidad Alberto Hurtado & 5 & 1 & Privada \\
\hline 13 & Universidad Austral de Chile & 5 & 2 & Privada, Consejo de Rectores \\
\hline 14 & Universidad de las Américas & 5 & 13 & Privada \\
\hline 15 & Universidad de los Andes & 5 & 2 & Privada \\
\hline 16 & Universidad de Valparaíso & 5 & 1 & Estatal, Consejo de Rectores \\
\hline 17 & Universidad del Biobío & 5 & 1 & Estatal, Consejo de Rectores \\
\hline 18 & Universidad Mayor & 5 & 2 & Privada \\
\hline 19 & Universidad del Desarrollo & 5 & 2 & Privada \\
\hline 20 & Universidad Andrés Bello & 4 & 3 & Privada \\
\hline 21 & Universidad Autónoma de Chile & 4 & 5 & Privada \\
\hline 22 & Universidad Católica del Maule & 4 & 1 & Privada, Consejo de Rectores \\
\hline 23 & Universidad Central de Chile & 4 & 4 & Privada \\
\hline 24 & Universidad de Tarapacá & 4 & 2 & Estatal, Consejo de Rectores \\
\hline 25 & Universidad de Viña del Mar & 4 & 2 & Privada \\
\hline 26 & Universidad San Sebastián & 4 & 5 & Privada \\
\hline 27 & Universidad Católica Silva Henríquez & 3 & 1 & Privada \\
\hline 28 & Universidad del Pacífico & 3 & 1 & Privada \\
\hline 29 & Universidad Santo Tomás & 3 & 19 & Privada \\
\hline 30 & Universidad Arturo Prat & 2 & 2 & Estatal, Consejo de Rectores \\
\hline
\end{tabular}




\begin{tabular}{llccl}
\hline & & Años & Progr. & \multicolumn{2}{c}{ Tipo de Universidad } \\
\hline 31 & Universidad Bernardo O’Higgins & 2 & 2 & Privada \\
32 & Universidad Academia de Humanismo Cristiano & NA & 2 & Privada \\
33 & Universidad Pedro de Valdivia & SA & 9 & Privada \\
34 & Universidad la República & SA & 2 & Privada \\
35 & Universidad de Artes, Ciencias y Comunicación & SA & 2 & Privada \\
36 & Universidad de Antofagasta & SA & 1 & Estatal, Consejo de Rectores \\
37 & Universidad de Atacama & SA & 1 & Estatal, Consejo de Rectores \\
38 & Universidad Adventista de Chile & SA & 1 & Privada \\
39 & Universidad de Magallanes & SA & 1 & Estatal, Consejo de Rectores \\
40 & Universidad ARCIS & SA & 2 & Privada \\
41 & Universidad de los Lagos & SA & 2 & Estatal, Consejo de Rectores \\
42 & Universidad de Aconcagua & SA & 17 & Privada \\
43 & Universidad Bolivariana & SA & 6 & Privada \\
44 & Universidad los Leones & SA & 2 & Privada \\
45 & Universidad Miguel de Cervantes & SA & 2 & Privada \\
46 & Universidad SEK & SA & 2 & Privada \\
47 & Universidad UCINF & SA & 2 & Privada \\
48 & Universidad Gabriela Mistral & SA & 1 & Privada \\
49 & Universidad Católica de Temuco & SA & 1 & Privada, Consejo de Rectores \\
\hline
\end{tabular}

NA: NO acredita; SA: No se han presentado a acreditación.

*El Consejo de Rectores de las Universidades Chilenas es una persona jurídica de derecho público, de administración autónoma, creado por ley el 14 de agosto de 1954, como un organismo de coordinación de la labor universitaria de la nación. Está integrado por los rectores de las veinticinco universidades públicas y tradicionales del país.

Fuente: elaboración propia

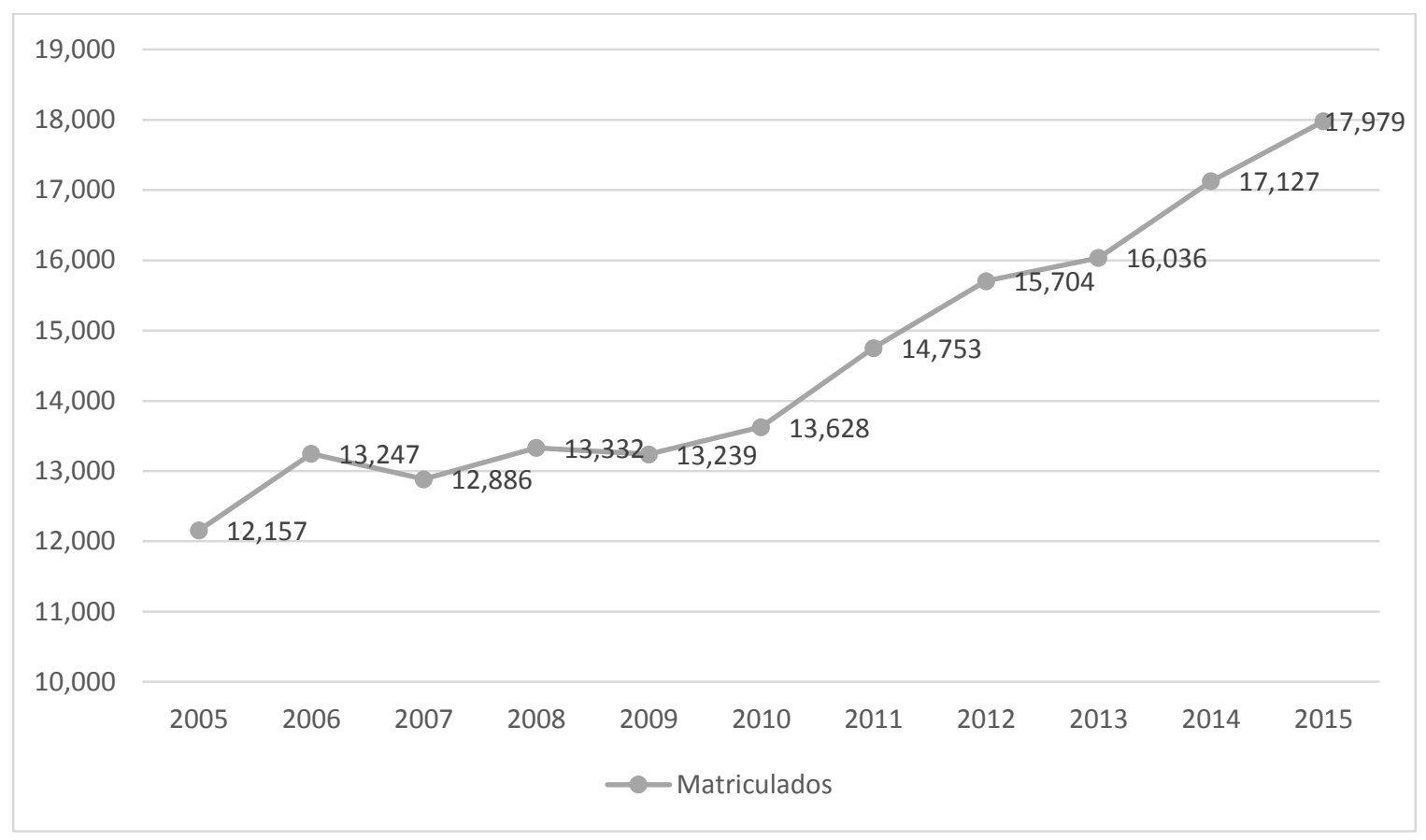

Gráfico 1. Número de estudiantes matriculados en programas de psicología 2005-2015.

Fuente: elaboración propia 


\section{Formación doctoral}

La formación doctoral en Chile es de reciente data. El año 1998 la Universidad de Chile (UCH) creó el primer programa de Doctorado en Psicología, sumándosele luego en el 2000 el programa de Doctorado en Psicología de la Pontificia Universidad
Católica (PUC) y en el 2007 el Doctorado Internacional en Psicoterapia, impartido en conjunto entre la UCH y la PUC con la Universidad de Heidelberg, Alemania (Urzúa, 2008). Actualmente, existe una oferta de nueve programas de formación doctoral en el país. Los programas vigentes, así como las líneas de investigación que informa cada

TABLA 2

Programas de doctorado vigentes en Chile y líneas de investigación respectivas a julio de 2015

\begin{tabular}{|c|c|c|c|}
\hline Universidad & $\begin{array}{l}\text { Programa } \\
\text { Doctorado }\end{array}$ & Líneas de Investigación & $\begin{array}{c}\text { Estado } \\
\text { acreditación }\end{array}$ \\
\hline $\begin{array}{l}\text { Pontificia Universidad } \\
\text { Católica de Chile }\end{array}$ & Psicología & $\begin{array}{l}\text { Procesos psicológicos en la enseñanza-aprendizaje } \\
\text { Psicología social y política } \\
\text { Psicología del desarrollo, relaciones interpersonales y vínculos } \\
\text { tempranos } \\
\text { Psicología de la salud } \\
\text { Salud mental y psicología clínica } \\
\text { Neurociencias y procesos psicológicos básicos }\end{array}$ & $\begin{array}{c}7 \text { años } \\
21 / 01 / 2009 \text { al } \\
21 / 01 / 2016\end{array}$ \\
\hline $\begin{array}{l}\text { Universidad de la } \\
\text { Frontera }\end{array}$ & Psicología & $\begin{array}{l}\text { Rol de la cultura en los procesos y disposiciones psicológicas y } \\
\text { el comportamiento }\end{array}$ & $\begin{array}{c}3 \text { años } \\
15 / 05 / 2013 \text { al } \\
15 / 105 / 2016\end{array}$ \\
\hline $\begin{array}{l}\text { Universidad de } \\
\text { Concepción }\end{array}$ & Psicología & $\begin{array}{l}\text { Psicología de los procesos de enseñanza y aprendizaje } \\
\text { Bienestar y salud }\end{array}$ & $\begin{array}{c}3 \text { años } \\
16 / 04 / 2014 \text { al } \\
16 / 04 / 2017\end{array}$ \\
\hline Universidad de Chile & Psicología & $\begin{array}{l}\text { Psicología experimental y neurociencia } \\
\text { Psicoanálisis y cultura } \\
\text { Psicología, educación y sociedad } \\
\text { Acción política e identidades sociales } \\
\text { Psicología clínica infanto-juvenil y sexualidad } \\
\text { Instituciones, organizaciones y vida cotidiana } \\
\text { Vulnerabilidades y desastres socionaturales }\end{array}$ & $\begin{array}{l}2 \text { años } \\
27 / 11 / 2013 \text { al } \\
27 / 11 / 2015\end{array}$ \\
\hline $\begin{array}{l}\text { Universidad de } \\
\text { Santiago de Chile }\end{array}$ & Psicología & $\begin{array}{l}\text { Ética e interculturalidad } \\
\text { Bienestar psicosocial y salud } \\
\text { Subjetividades y género } \\
\text { Psicología cultural y política } \\
\text { Psicología evolucionaria: afectividad y relaciones } \\
\text { interpersonales. }\end{array}$ & No acreditado \\
\hline $\begin{array}{l}\text { Pontificia Universidad } \\
\text { Católica de Valparaíso }\end{array}$ & Psicología & $\begin{array}{l}\text { Análisis de los procesos psicológicos implicados en las } \\
\text { transformaciones sociales de carácter institucional en: } \\
\text { Educación } \\
\text { Trabajo y organizaciones } \\
\text { Salud y desarrollo comunitario }\end{array}$ & No acreditado \\
\hline $\begin{array}{l}\text { Universidad Diego } \\
\text { Portales }\end{array}$ & Psicología & $\begin{array}{l}\text { Procesos psicosociales: sujetos, discursos y prácticas sociales } \\
\text { Psicoanálisis: clínica, sociedad y cultura } \\
\text { Neurociencia, cognición social y procesos decisionales }\end{array}$ & $\begin{array}{l}\text { Programa } \\
\text { nuevo sin } \\
\text { acreditar }\end{array}$ \\
\hline $\begin{array}{l}\text { Universidad Católica } \\
\text { del Norte }\end{array}$ & Psicología & $\begin{array}{l}\text { Género y Sexualidad } \\
\text { Salud Mental y contextos relacionales } \\
\text { Salud, bienestar y calidad de vida }\end{array}$ & $\begin{array}{l}\text { Programa } \\
\text { nuevo sin } \\
\text { acreditar }\end{array}$ \\
\hline $\begin{array}{l}\text { Pontificia Universidad } \\
\text { Católica de Chile }\end{array}$ & Psicoterapia & Psicoterapia y etiología clínica intercultural & $\begin{array}{l}5 \text { años } \\
11 / 07 / 2012 \text { al } \\
11 / 07 / 2017\end{array}$ \\
\hline
\end{tabular}

Fuente: elaboración propia

\begin{tabular}{|l|l|l|l|l|} 
Universitas Psychologica & V. 14 & No. 3 & Julio-septiembre & 2015 \\
\hline
\end{tabular} 
uno de ellos y el estado de acreditación, se pueden observar en la Tabla 2, y el desarrollo histórico de la formación doctoral en Chile se puede revisar en Vera-Villarroel (2010).

Un hecho destacable es que parte de la formación doctoral en el país es financiada directamente por la Comisión Nacional de Investigación Científica y Tecnológica (CONICYT) del Gobierno de Chile, organismo creado el año 1967 y cuyos pilares estratégicos son, por un lado, fortalecer el capital humano avanzado a través de la entrega de becas para cursar estudios de postgrado, y por otra parte el desarrollo y fortalecimiento de la base científica y tecnológica (CONICYT, 2015). En los últimos cinco años, 120 estudiantes han sido financiados por CONICYT para cursar estudios doctorales en programas chilenos en psicología (Tabla 3). Cabe mencionar que un requisito para optar a estas becas es que el programa de doctorado al que se postula esté acreditado.

CONICYT igualmente financia estudios en el extranjero para cursar maestrías y doctorados.

TABLA 3

Estudiantes de doctorado en Chile financiados por el Gobierno de Chile, periodo 2011-2015, por programa de doctorado

\begin{tabular}{lcccccc}
\hline Programa Doctoral & 2011 & 2012 & 2013 & 2014 & 2015 & TOTAL \\
\hline Psicología U. de Chile & 8 & 11 & 8 & 5 & 8 & 40 \\
\hline Psicología Pontificia U. Católica & 2 & 7 & 6 & 9 & 11 & 35 \\
\hline Psicología U. de Concepción & & 2 & 1 & 1 & 3 & 7 \\
\hline Psicología U. de la Frontera & & & 2 & 1 & 0 & 3 \\
\hline Psicología U. de Santiago de Chile & 4 & 3 & 5 & 2 & 10 & 24 \\
\hline Psicoterapia PUC/UCH & & & & 4 & 4 & 8 \\
\hline Salud Mental U. de Concepción & & & 3 & & 3 \\
\hline Neurociencia Pontificia U. Católica & 14 & 23 & 22 & 32 & 41 & 132 \\
\hline Ciencias m/Neurociencia U. Valparaíso & & & & & & \\
\hline TOTAL & & & & & & \\
\hline
\end{tabular}

Fuente: elaboración propia

TABLA 4

Estudiantes de doctorado en el extranjero financiados por el Gobierno de Chile, periodo 2011-2015, por área de la psicología

\begin{tabular}{lc}
\hline Área de la psicología & N. ${ }^{\circ}$ becarios \\
\hline Neurociencias/neuropsicología & 12 \\
\hline Psicología & 11 \\
\hline Psicología clínica/personalidad & 10 \\
\hline Psicología de la salud / psicología médica & 6 \\
\hline Psicología social & 6 \\
\hline Psicología del desarrollo & 5 \\
\hline Cs. sociales /sociología /ciencias políticas & 5 \\
\hline Investigación y metodología & 3 \\
\hline Psicología y educación & 3 \\
\hline Criminología & 3 \\
\hline Psicoanálisis & 2 \\
\hline Psicología Experimental & 1 \\
\hline TOTAL & 67 \\
\hline
\end{tabular}

Fuente: elaboración propia 
Entre los años 2011 y 2015 se han financiado a 67 estudiantes para realizar estudios doctorales en distintas partes del mundo, principalmente en el campo de las neurociencias, por lo que se espera un aumento en la productividad científica asociada a esta área, en el plazo cercano. El número de becarios por área general de la psicología se puede observar en la Tabla 4.

\section{Productividad científica}

Para las siguientes tablas, se consideraron las publicaciones en la base de datos WOS (ex ISI) con dirección "Chile" entre los años 2005 y 2014. De ellas se analizaron solo las clasificadas en Psicología (Psychology). Dado que el objetivo de este artículo es mostrar las tendencias generales, solo se analizó esta categoría. Estudios más especializados en investigación podrán analizar especificaciones y áreas especializadas de la disciplina. Los resultados mostraron un total de 984 documentos.

Como se puede observar en el Gráfico 2, existe un alza sostenida en la cantidad de artículos publi- cados, pasando de 32 artículos en el año 2005 a 188 en el 2014. Se destaca que a partir del año 2011 se sobrepasan los 100 artículos por año.

Con respecto a las instituciones chilenas responsables de los artículos analizados, destacan considerablemente cuatro instituciones por sobre el resto. La Pontificia Universidad Católica de Chile es la primera universidad con 342 artículos, duplicando a la segunda institución que es la Universidad de Chile. Esto es coherente con los análisis de la productividad de las instituciones chilenas donde ambas universidades sobresalen en los primeros lugares de Chile en todas las ciencias (Vera-Villarroel, López-López, Lillo, \& Silva, 2011). Posteriormente, se sitúan la Universidad Diego Portales y la Universidad de Santiago con 97 y 89 artículos, respectivamente. Le sigue un segundo grupo de universidades que van de 55 artículos a 31: Universidad de Talca, Concepción, Católica del Norte, Desarrollo y Frontera.

En la Tabla 5, se presentan las primeras 15 instituciones según productividad, sin embargo, estos datos deben analizarse con precaución. En algunas más complejas es habitual que el sistema

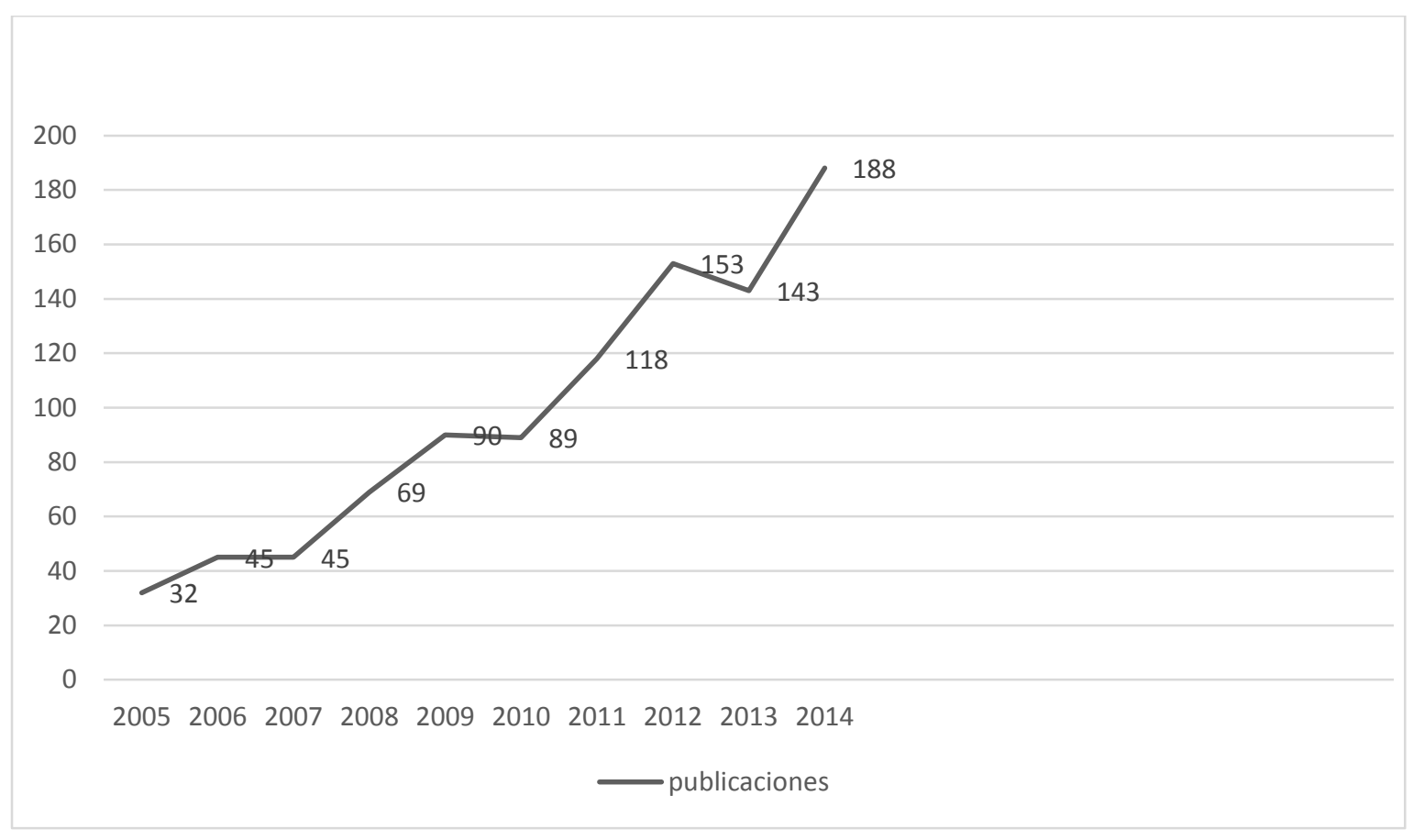

Gráfico 2. Número de publicaciones en psicología 2005-2014.

Fuente: elaboración propia 
clasifique artículos en la categoría de psicología, aun cuando los autores no correspondan a las escuelas o facultades de psicología. Esto es especialmente significativo en la cantidad de artículos asignados en la Universidad de Chile, donde un número considerable corresponde a autores de Facultades o carreras de Medicina, Psiquiatría o Biología. Debe considerarse además el caso de entidades que pueden aparecer con poca productividad en psicología, a pesar de que sus investigadores estén contribuyendo activamente en áreas de otras disciplinas científicas y que no son recogidas en revistas de psicología (p. ej., revistas médicas, de salud pública o de ciencias sociales). Mención aparte merece también la productividad de una institución que descansa principalmente en la productividad de un solo investigador.

Con respecto a los autores más productivos, se distingue un autor con 43 artículos, posteriormente un grupo de autores entre los 26 y 20 artículos. Luego los autores más productivos fluctúan entre los 17 y 10 artículos. Es necesario comentar la situación del primer autor que con 43 artículos es responsable del $44 \%$ del total de su institución (Universidad Diego Portales) ya que el investigador publica (y por lo tanto incrementa la producti- vidad) con más de una filiación institucional, y en algunos de sus artículos se presenta con filiación de varias instituciones y de varios países diferentes. Se debe tener presente que los investigadores en psicología también publican habitualmente en revistas de otras especialidades, ejemplo, educación, medicina, neurociencias o economía, entre otras, productividad que no ha sido contemplada en esta oportunidad (Tabla 6).

Con respecto al idioma de los artículos, se denota que 611 de estos (62\%) están escritos en inglés y solo 348 (35\%) en español. Se supondría que los autores chilenos publicaran en primera instancia en el idioma español, pero como se observa esto no ocurre.

Con respecto a las revistas en las que más publican autores chilenos, resaltan en la tabla Terapia Psicológica y Universitas Psychologica. Le siguen dos revistas latinoamericanas como son la Revista Argentina de Clínica Psicológica y la Revista Latinoamericana de Psicología, ambas con 51 artículos, y posteriormente dos revistas españolas y por último aparecen revistas angloparlantes. El listado de artículos en las primeras 30 revistas con mayor frecuencia de publicaciones se puede observar en la Tabla 7.

\section{TABLA 5}

Número de artículos publicados en psicología por universidad periodo 2005- 2014

\begin{tabular}{clc}
\hline & Instituciones & N. ${ }^{\circ}$ artículos \\
\hline 1. & Pontificia Universidad Católica de Chile & 342 \\
2. & Universidad de Chile & 156 \\
3. & Universidad Diego Portales & 97 \\
4. & Universidad de Santiago de Chile & 89 \\
5. & Universidad de Talca & 55 \\
6. & Universidad de Concepción & 54 \\
7. & Universidad Católica del Norte & 50 \\
8. & Universidad del Desarrollo & 40 \\
9. & Universidad de la Frontera & 38 \\
10. & Pontificia Universidad Católica de Valparaíso & 31 \\
11. & Universidad de Tarapacá & 28 \\
12. & Universidad Adolfo Ibáñez & 23 \\
13. & Universidad de Valparaíso & 23 \\
14. & Universidad Alberto Hurtado & 18 \\
15. & Universidad Santo Tomás & 12 \\
\hline
\end{tabular}

Fuente: elaboración propia 
Con respecto a las subcategorías de los artículos, sobresale en primer lugar psicología multidisciplinaria y en segundo lugar Psicología Clínica. Le siguen un grupo de áreas de neurociencias y Psicología Experimental. Posteriormente, Psicología Social y más atrás Psicología Educacional (Tabla 8).

En la Tabla 9, se pueden apreciar los países con los cuales colaboran autores chilenos, destacando en primer lugar a Estados Unidos, seguidos de España e Inglaterra. Llama la atención la escasa colaboración con autores latinoamericanos, aspecto ya identificado como un problema regional en estudios previos (García, Acevedo-Triana, \& López-López, 2014), apareciendo solo Argentina en el cuarto lugar y Colombia en el décimo. Posteriormente, Brasil en el décimo quinto y México en el décimo noveno.

\section{Asociatividad}

Una de las áreas más precarias en Chile es la pertenencia y participación en sociedades y asociaciones. Luego del inicio de la dictadura militar en el año 1973, los colegios profesionales perdieron la obligatoriedad de colegiatura, transformándose en aso-

TABLA 6

Número de artículos publicados en psicología por autor periodo 2005-2014, con 10 o más artículos

\begin{tabular}{lll}
\hline & Autores & N.o artículos \\
\hline 1 & Ibáñez, A.* & 43 \\
2 & González, R. & 26 \\
3 & Vera-Villarroel, P. & 26 \\
4 & Manes, F. & 23 \\
5 & López, V. & 20 \\
6 & Cornejo, C. & 17 \\
7 & Farkas, Ch. & 16 \\
8 & Santelices, M. P. & 16 \\
9 & Urzúa, A. & 16 \\
10 & Cumsille, P. & 15 \\
11 & Fernández, A. M. & 15 \\
12 & Krause, M. & 15 \\
13 & Tomicic, A. & 15 \\
14 & Haye, A. & 13 \\
15 & Hurtado, E. & 13 \\
16 & Jiménez, J. P. & 13 \\
17 & Laborda, M. & 13 \\
18 & Valdés, N. & 12 \\
19 & Aboitiz, F. & 11 \\
20 & Alcalay, L. & 11 \\
21 & Berger, C. & 11 \\
22 & Caqueo-Urízar, A. & 11 \\
23 & Celis-Atenas, K. & 11 \\
24 & Cosmelli, D. & 11 \\
25 & Martínez, M. L. & 11 \\
26 & Strasser, K. & 11 \\
27 & Huepe, D. & 10 \\
28 & Vogel, E. & 10 \\
\hline
\end{tabular}

*Autor extranjero con alguna de sus filiaciones correspondiendo a una universidad chilena.

Fuente: elaboración propia 
TABLA 7

Número de artículos de psicología publicados por revista: periodo 2005-2014

\begin{tabular}{clcc}
\hline & \multicolumn{1}{c}{ Revistas } & N. ${ }^{\circ}$ de artículos & $\%$ \\
\hline 1 & Terapia Psicológica & 91 & 9.362 \\
2 & Universitas Psychologica & 91 & 9.362 \\
3 & Revista Argentina de Clínica Psicológica & 51 & 5.247 \\
4 & Revista Latinoamericana de Psicología & 51 & 5.247 \\
5 & Estudios de Psicología & 25 & 2.572 \\
6 & Psicothema & 23 & 2.366 \\
7 & Frontiers in Human Neuroscience & 21 & 2.16 \\
8 & International Journal of Psychology & 21 & 2.16 \\
9 & Ethology & 19 & 1.955 \\
10 & Anales de Psicología & 17 & 1.749 \\
11 & Psychophysiology & 14 & 1.44 \\
12 & Computers in Human Behavior & 13 & 1.337 \\
13 & Revista Mexicana de Psicología & 12 & 1.235 \\
14 & Culture Psychology & 11 & 1.132 \\
15 & Integrative Psychological and Behavioral Science & 11 & 1.132 \\
16 & International Journal of Psychoanalysis & 11 \\
17 & Spanish Journal of Psychology & 11 & 1.132 \\
18 & Frontiers in Psychology & 11 & 1.132 \\
19 & International Journal of Clinical and Health Psychology & 9 & 0.926 \\
20 & Annals of Behavioral Medicine & 9 & 0.926 \\
21 & Cyberpsychology Behavior & 8 & 0.823 \\
22 & Journal of Cross Cultural Psychology & 8 & 0.823 \\
23 & International Psychogeriatrics & 8 & 0.823 \\
24 & Revista de Psicologia Social & 7 & 0.72 \\
25 & Behavioural Processes & 7 & 0.72 \\
26 & Infancia y Aprendizaje & 6 & 0.617 \\
27 & Infant Mental Health Journal & 6 & 0.617 \\
28 & Psicologia Reflexao e Critica & 6 & 0.617 \\
29 & Psychological Medicine & 6 & 0.617 \\
30 & Revista de Psicología del Deporte & 6.617 \\
\hline & ent & 6.617 \\
\hline
\end{tabular}

Fuente: elaboración propia

TABLA 8

Número de artículos publicados en psicología por categoría periodo 2005-2014

\begin{tabular}{llccc}
\hline & & Categorías & N. ${ }^{\circ}$ artículos & $\%$ \\
\hline 1 & Psychology Multidisciplinary & 373 & 38.37 \\
2 & Psychology Clinical & 222 & 22.84 \\
3 & Psychology & 169 & 17.38 \\
4 & Psychology Experimental & 93 & 9.56 \\
5 & Neuroscieces & 89 & 9.15 \\
6 & Psychology Biological & 72 & 7.4
\end{tabular}




\begin{tabular}{clcc}
\hline & \multicolumn{1}{c}{ Categorías } & N. ${ }^{\circ}$ artículos & $\%$ \\
\hline 7 & Psychology Social & 69 & 7.09 \\
8 & Behavioral Sciences & 64 & 6.58 \\
9 & Psychology Developmental & 61 & 6.27 \\
10 & Psychiatry & 41 & 4.21 \\
11 & Psychology Educational & 37 & 3.8 \\
12 & Psychology Applied & 35 & 3.6 \\
13 & Zoology & 30 & 3.08 \\
14 & Psychology Psychoanalysis & 23 & 2.36 \\
15 & Physiology & 18 & 1.85 \\
16 & Social Sciences Interdisciplinary & 18 & 1.852 \\
17 & Public Environmental Occupational Health & 16 & 1.64 \\
18 & Communication & 15 & 1.54 \\
19 & Psychology Mathematical & 15 & 1.54 \\
20 & Sociology & 13 & 1.33 \\
\hline
\end{tabular}

Fuente: elaboración propia

\section{TABLA 9}

Número de artículos publicados en colaboración por país periodo 2005-2014

\begin{tabular}{llll}
\hline & País & N. ${ }^{\circ}$ artículos & $\%$ \\
\hline 1 & Estados Unidos & 172 & 17.695 \\
2 & España & 163 & 16.77 \\
3 & Inglaterra & 68 & 6.996 \\
4 & Argentina & 55 & 5.658 \\
5 & Alemania & 48 & 4.938 \\
6 & Francia & 30 & 3.086 \\
7 & Italia & 29 & 2.984 \\
8 & Australia & 28 & 2.881 \\
9 & Bélgica & 28 & 2.881 \\
10 & Colombia & 26 & 2.675 \\
11 & Holanda & 24 & 2.469 \\
12 & Suiza & 20 & 2.058 \\
13 & Canadá & 19 & 1.955 \\
14 & Portugal & 18 & 1.852 \\
15 & Brasil & 17 & 1.749 \\
16 & Japón & 16 & 1.646 \\
17 & China & 15 & 1.543 \\
18 & Estonia & 15 & 1.44 \\
19 & México & 14 & 1.44 \\
20 & Nueva Zelanda & 14 & 1.337 \\
\hline
\end{tabular}

Fuente: elaboración propia 
ciaciones gremiales de libre asociación. Este hecho perdura hasta el día de hoy. En los últimos 10 años ha habido un aumento en el número de asociaciones y sociedades en Chile, la mayoría vinculadas a especialidades, sobresaliendo la formación de la Sociedad Científica de Psicología de Chile, en el año 2011, la que se ha incorporado al Consejo de Sociedades Científicas de Chile. El listado de organizaciones conocidas se puede ver en la Tabla 10.

\section{Desafíos}

El gran incremento en el número de programas de pregrado en psicología, así como su calidad dispar, observable a través de los resultados de los procesos de acreditación, dejan en claro que uno de los más importantes desafíos de la psicología chilena es asegurar la calidad de la formación de los profesionales psicólogos.

En este contexto de extensa oferta de programas y ausencia de un mecanismo eficaz de acompañamiento a los postulantes, que facilite su discernimiento más allá de la inversión en marketing que puedan hacer las distintas instituciones, se hace imprescindible un esfuerzo colectivo para presionar a los legisladores y al Estado para lograr que la carrera de Psicología sea de acreditación obligatoria. Sin este mínimo resguardo de la calidad de la formación, estamos en grave falta, no solo frente a los propios estudiantes, sino frente a la sociedad en su conjunto, ya que como disciplina debiéramos ser capaces de asegurar que los psicólogos que prestan servicios en nuestras comunidades cuenten con las competencias mínimas requeridas, en términos de formación ética, científica y profesional.

El necesario aseguramiento de la calidad debe implicar no tan solo la formación inicial, sino también la formación posgraduada, debiendo ser también obligatoria la acreditación de los programas de maestría y doctorado, ya que en la actualidad está vinculada únicamente a la posibilidad del estudiante de solicitar recursos del Estado a través de becas, únicamente cuando el programa está acreditado y a que, tal como se ha mencionado, pese a que existe un sistema de acreditación, y este es actualmente voluntario en el caso de la psicología.

TABLA 10

Organizaciones científicas y gremiales de psicología en Chile a junio de 2015

\begin{tabular}{|c|c|}
\hline Organización & Página web \\
\hline Colegio de Psicólogos de Chile $^{1}$ & http://www.colegiopsicologos.cl/ \\
\hline Sociedad Científica de Psicología de Chile ${ }^{2}$ & http://cienciapsicologica.cl/ \\
\hline Sociedad Chilena de Psicología Clínica ${ }^{3}$ & http://www.scpc.cl/ \\
\hline Sociedad Chilena de Medicina conductual y Psicología de la Salud ${ }^{3}$ & http://www.sch-mc-ps.cl/ \\
\hline Sociedad Chilena de Psicología Organizacional ${ }^{3}$ & http://schipo.blogspot.cl/ \\
\hline Asociación Nacional de Psicólogos Educacionales ${ }^{3}$ & http://www.anpse.blogspot.cl/ \\
\hline Sociedad Chilena de Psicología en Emergencia y Desastres ${ }^{3}$ & http://www.sochped.cl/ \\
\hline Sociedad Chilena de Psicología Analítica ${ }^{3}$ & http://www.cgjungchile.cl/ \\
\hline Sociedad Chilena de Psicología Comunitaria ${ }^{3}$ & Sin página \\
\hline Sociedad Chilena de Psicología del Deporte ${ }^{3}$ & http://www.emd.cl/sochipd.cl/ \\
\hline Sociedad Chilena de Psicología Positiva ${ }^{3}$ & http://www.sochipsp.cl/ \\
\hline Asociación Chilena de Psicología Positiva ${ }^{3}$ & http://acpp.cl/ \\
\hline Asociación Chilena de Psicología Jurídica y Forense ${ }^{3}$ & http://www.asociacionpsicologiajuridica.cl/ \\
\hline Sociedad Chilena de Historia de la Psicología & Sin página \\
\hline
\end{tabular}

${ }^{1}$ De carácter gremial; requisito de afiliación: poseer el título de psicólogo; ${ }^{2}$ asociación científica; requisito de afiliación: estar en posesión del grado de doctor/a y tener una línea de investigación vigente, o estar cursando un programa doctoral; ${ }^{3}$ asociación científica; requisito de afiliación: ser psicólogo con desempeño en el área, exceptuando Medicina conductual que es interdisciplinaria.

Fuente: elaboración propia 
Esta necesidad de modificar la legislación, nos lleva a otro de nuestros principales desafíos, que es mejorar nuestra capacidad de influir en la opinión pública. Nuestra presencia en el debate legislativo es claramente insuficiente, a pesar de que todas las reformas que se están desarrollando actualmente en nuestro país atañen directamente al objeto de estudio de la psicología, como son la educación, la familia, la salud, el trabajo, entre otros. Es necesario contar con psicólogos que asuman roles protagónicos en los debates e implementación de iniciativas estatales y privadas. Podría concluirse que existe en esto un ligero progreso, ya que en el actual gobierno se cuenta con dos ministros de Estado que son psicólogos, sin embargo, la presencia pública de la psicología es aún insuficiente.

Para mejorar la capacidad de influencia de la psicología se requiere, por una parte, colaborar con los medios de comunicación, para promover la difusión del conocimiento psicológico y su puesta al servicio del desarrollo y bienestar de la ciudadanía y, por otro lado, mejorar de la capacidad de asociatividad de los psicólogos de modo que puedan trabajar conjuntamente, por una meta que trascienda intereses gremiales, personales e institucionales.

Los planes de formación en psicología, antiguos y actuales, parecen no estimular adecuadamente valores como la asociatividad y el trabajo colaborativo. Se necesita apelar a los propios conocimientos desarrollados por la disciplina para lograr cambiar esta situación y motivar a los nuevos profesionales y científicos a formar parte de un esfuerzo colectivo. En un contexto en que las formas tradicionales de participación -participación electoral, militancia partidista, involucramiento en campañas, etc.- parecen irse debilitando (Putnam, 2000), el desafío es aún mayor.

En este esfuerzo por construir una comunidad organizada y comprometida, la psicología chilena requiere además, establecer alianzas con organizaciones de profesionales y científicos de otras disciplinas, para sostener la defensa del conocimiento basado en la evidencia y demandar un apoyo decidido por parte del Estado a la investigación científica en los distintos ámbitos del conocimiento.
El aumento en becas para estudios de doctorado en psicología, tanto en Chile como en el extranjero, aunque notable, aún no se traduce en un crecimiento de igual magnitud en la productividad de la disciplina, pues aunque ha ido claramente en aumento, este aún es insuficiente para posicionar al país como un polo de desarrollo del conocimiento psicológico en el contexto iberoamericano. Para que se produzca el despegue esperado, se debe incrementar la capacidad de colaboración y el apoyo en recursos a los programas doctorales nacionales. Especial desafío plantea la necesidad de construir alianzas con investigadores latinoamericanos, con quienes se comparte no solo el idioma, sino también muchos de nuestros principales problemas y particularidades. Debemos ser capaces, además, de desarrollar el trabajo interdisciplinario, para poder dar cuenta en mejor medida de la complejidad de los fenómenos estudiados.

Con el notorio incremento en el número de profesionales psicólogos que se titula cada año, la psicología chilena se enfrenta al mismo tiempo a un problema y una oportunidad. Una forma de abordar la situación es concluir que el campo laboral se estaría ya saturando y que es necesario limitar el acceso y el número de programas. Otra opción es asumir el desafío de ampliar el área de desempeño de los psicólogos a la vez que idear sistemas de reconocimiento y acreditación de las diversas especialidades de la psicología, dado que hasta el momento la única acreditación profesional se hace en el ámbito de la psicología clínica.

Como señala el expresidente de la APA, James Bray (2009), la mayoría de las personas tienen la creencia de que el psicólogo es únicamente un profesional de la salud mental, sin embargo, para tener éxito en el futuro, los psicólogos tenemos que ampliar nuestra visión de lo que constituye la investigación y la práctica psicológica. Debemos reconocer oportunidades y pensar globalmente acerca de los roles que la psicología puede desempeñar en el siglo XXI. Uno de estos caminos es poner los esfuerzos en la prevención y no solo en la intervención, y el especial rol que deben jugar los psicólogos en estos esfuerzos, ya sea en el campo de la salud, la edu- 
cación, el trabajo y las organizaciones, el derecho, entre muchos otros.

Otros componentes fundamentales por considerar son el multiculturalismo y la internacionalización. Desafíos que quiéralo o no, el psicólogo se verá obligado a enfrentar. El creciente aumento de inmigrantes y refugiados, sumado a un creciente reconocimiento de los derechos de los pueblos originarios, dan cuenta de esta necesidad. La creciente población de adultos mayores y la mayor visibilización de minorías sexuales y personas con discapacidad física o mental, evidencian también otras problemáticas que la psicología deberá abordar. La formulación de políticas, el mundo de los negocios y del marketing, la salud pública, las comunidades, las organizaciones sociales, las tecnologías y el bienestar son espacios con un amplio potencial de desarrollo.

En definitiva, la práctica psicológica en el Chile del siglo XXI requiere que cambiemos nuestras formas tradicionales de entender la psicología, para aprovechar las nuevas posibilidades que nos brindan los vertiginosos cambios sociales.

\section{Referencias}

Alarcón, R. (1997). Orientaciones teóricas de la psicología en América Latina. Lima: Universidad Femenina del Sagrado Corazón.

Alarcón, R. (2002). Estudios sobre psicología latinoamericana. Lima: Editorial Universitaria.

Alfonso, A., \& Pacheco, S. (2011). Elisabeth Isabel Bongard. Migrante y protagonista de la reforma educacional alemana en Chile. La Serena: Editorial Universidad de La Serena.

Barrientos, J., Palma, I., \& Gómez, F. (2014). Discursos sobre sexología en Chile. Ambivalencias del discurso profesional sobre la sexología en Chile. Terapia Psicológica, 32(2), 101-110.

Bravo, L. (2004). Cincuenta años de psicología en la Universidad Católica. Psykhé, 13(1), 97-204.

Bray, J. H. (febrero, 2009). Vision for the future of psychology. Monitor on Psychology, 40(2), 5. Disponible en http://www.apa.org/monitor/2009/02/ pc.aspx
Calderon, R. (2000). Antecedentes sobre el rol del psicólogo y su formación. En: G. Miranda, R. Calderón \& X. Bugueño (Eds). Psicología contemporánea: Disciplina, profesión, formación (pp. 51-106). Santiago: Universidad Católica Cardenal Raúl Silva Henriquez.

Colegio de Psicólogos de Chile. (1985). Análisis del primer Congreso Nacional de Psicólogos. Psicología y Calidad de Vida. Santiago: Autor.

Comisión Nacional Científica y Tecnológica. (2015). Página oficial del Gobierno de Chile. Disponible en http://www.conicyt.cl

Comisión Nacional de Acreditación. (2015). Página oficial del Gobierno de Chile. Disponible en https:// www.cnachile.cl

Consejo Nacional de Educación. (2015). Página oficial del Gobierno de Chile. Disponible en http://www. cned.cl

Cubillos, L., \& Agüero, G. (1941). Higiene mental y psiquiatría. Santiago: Sin editorial.

Descouvieres, C. (1985). El Colegio de Psicólogos. Ente regulador de la profesión. En Anales del primer Congreso Nacional de Psicólogos (pp. 143-146). Santiago: Colegio de Psicólogos de Chile.

Descouvieres, C. (1999). Lo psicológico en los Anales de la Universidad de Chile. Santiago: Universitaria.

Foradori, A. (1954). La psicología en América. Buenos Aires: Instituto Cultural Joaquín González.

García, A., Acevedo-Triana, C. A., \& López-López, W. (2014). Cooperación en las Ciencias del Comportamiento Latinoamericanas: una investigación documental. Terapia Psicológica, 32(2), 165-174.

Hanisch, W. (1963). En torno a la filosofía en Chile (15941810). Santiago: Universidad Católica de Chile.

Instituto de Chile. (1982). Homenaje a Don Andrés Bello. Santiago: Editorial Jurídica de Chile.

Iturriaga, A. (1944). Archivos del Instituto Central de Psicología. Santiago: Universitaria.

Horvitz, I. (1946). La necesidad de una legislación sobre enfermos mentales. Revista de Ciencias Penales, 9(2-3), 109-118.

Jaksic, I. (2001). Andrés Bello: la pasión por el orden. Caracas: Universidad Católica Andrés Bello.

Juliá, M. T. (Ed.). (2013). Competencias del psicólogo en Chile. Propuestas desde las universidades estatales. La Serena: Editorial Universidad de La Serena. 
Kalawski, A., Sepúlveda, P., \& Célis, A. (1996). La psicoterapia humanista y transpersonal: orígenes y conceptos fundamentales. En A. Célis (Ed.), Humanismo, espiritualidad y psicoterapia (pp. 19-30). Santiago: Transformación.

Letelier, V. (1927). Filosofía de la educación. Buenos Aires: Cabaut y Cia. Editores.

Ligueño, S., Parra, D., Moncada, L., \& Laborda, M. (2010). La psicología en la Universidad de Chile. Antecedentes históricos y trayectoria de la carrera en el período 1947-1992. En M. A. Laborda \& V. Quezada (Eds.), Notas históricas de la psicología en Chile (pp. 27-56). Santiago: Universitaria.

Lillo, S., \& Martini, N. (2013). Principales tendencias iberoamericanas en psicología clínica. Un estudio basado en la evidencia científica. Terapia Psicológica, 31(3), 363-371.

Loubat, M. (2013). La oferta del título de psicólogo en Chile: una aproximación justificativa crítica. En M. T. Juliá (Ed.), Competencias del psicólogo en Chile. Propuestas desde las universidades estatales (pp. 45-81). La Serena: Editorial Universidad de La Serena.

Luco, A. (2010). Psicología en Chile. Ciencia, disciplina y profesión. Santiago: Catalonia.

Mansur, M., \& Nóbrega, E. (1999). Una aproximación al desarrollo de la psicología en Chile. En C. Di Doménico \& A. Vilanova (Eds.), Formación de psicólogos en el Mercosur (pp. 65-75). Mar del Plata: UNMP.

Miguez, G., Betancourt, R., \& Vera-Villarroel, P. (2010). Historia de los laboratorios de psicología en Chile. En M. A. Laborda \& V. Quezada (Eds.), Notas históricas de la psicología en Chile (pp. 81-104). Santiago: Universitaria.

Morales, M., Díaz, R., Scharager, J., \& Sziklai, G. (1988). La formación profesional de los psicólogos en Chile. Análisis de la opinión de psicólogos y estudiantes de la carrera. Revista Chilena de Psicología, 9(1), 31-42.

Munizaga, F. (1967). Geocronología de los esquistos del sur de Chile. Jornadas de Trabajo, Resúmenes, Inst. Invest. Geol, 36-37.

Munizaga, R., \& Cizaletti, J. (1967). Vida y obra de Abelardo Iturriaga. Santiago: Editorial Universitaria.
Chile, Decreto con toma de razón N. 0170 de 2009 [Ministerio de Educación]. Fija normas para determinar los alumnos con necesidades educativas especiales que serán beneficiarios de las subvenciones para educación especial. Mayo 14 de 2009.

Nassar, C. (1955). Experiencias recogidas en torno a la preparación profesional del psicólogo en Chile. En Sociedad Interamericana de Psicología. Actas del Primer Congreso Interamericano de Psicología (pp. 571-586). Ciudad Trujillo: Editora del Caribe.

Parra, D. (2015). Un análisis historiográfico sobre las relaciones entre psicología y educación en Chile (1889-1973). Revista de Historia de la Psicología, 36(2), 95-114.

Pizarro, C. (1999). Imprecisiones en los recuentos históricos de la psicología chilena. Revista Chilena de Psicología, 18(2), 53-57.

Poblete, M. (1980). A propósito del centenario de la Creación del Primer Laboratorio de Psicología Experimental. Revista Chilena de Psicología, 3(1), 15-19.

Poblete, M. (1995). Raíces de la psicología experimental en el mundo iberoamericano. Revista Historia de la Psicología, 1(2), 255-262.

Putnam, R. (2000). Bowling alone. The collapse and revival of American community. New York: Simon $\&$ Schuster.

Quezada, V., Vergés, A., \& Laborda, M. (2014). Sergio Yulis: pasado y presente del enfoque conductual en Chile. Psykhé, 23(1), 1- 11.

Rodríguez, M., \& Villegas, J. F. (2007). Historia de la investigación científica de la psicología en Chile (Vol. 2). Santiago: Universidad Central de Chile.

Rosales, E. (27 de marzo de 1955). [Carta dirigida a R. P. Agustín Martínez]. Archivo Pontificia Universidad Católica de Chile.

Ruperthuz, M. (2012).The return of the repressed. The rol of sexuality in the reception of psychoanalysis in Chilean medical circles (1910s-1940s). History EO Psychoanalysis, 2, 285-296.

Salas, G. (2014a). El informe Nassar (1955) sobre la formación de psicólogos en Chile. Revista de Psicología, 23(1), 109-112.

Salas, G. (2014b). Pasado y presente de la psicología en Chile: profesionalización, instituciones y divulgación científica. En G. Salas (Ed.), Historias de la 
psicología en América del Sur. Diálogos y perspectivas (pp. 95-114). La Serena: Nueva Mirada.

Salas, G. (2012). La influencia europea en los inicios de la historia de la psicología en Chile. Interamerican Journal of Psychology, 46(1), 99-110.

Salas, G., \& Inzunza, J. (2013). Antecedentes históricos de la psicología educacional en Chile. En C. Cornejo, P. Morales, E. Saavedra \& G. Salas. Aproximaciones en psicología educacional (pp. 2741). Talca: UCM.

Salas, G., \& Lizama, E. (2013). Historia de la psicología en Chile. 1889-1981 (2. ${ }^{\text {a }}$ ed.). La Serena: Editorial Universidad de La Serena.

Tirapegui, L. (1928). El desarrollo de la inteligencia medido por el Test Binet-Simon. Santiago: Ministerio de Educación.

Urra, M. (2008). Asociación Chilena para la Formación Académica y Profesional en Psicología. En ACHIFAPS. Primer Seminario Nacional: Formación en Psicología en Chile, 1, 13-17.

Urzúa, A. (2008). Elementos contextuales a la investigación en psicología en Chile. Revista Interamericana de Psicología, 42(3), 446-461.

Venturino, A. (1929). Sociología chilena con comparaciones argentinas y mejicanas. Barcelona: Cervantes.
Vera-Villarroel, P. (2010). Historia y estado actual de la formación doctoral en Chile. Revista Digital Universitaria, 11(5), 1067-1079.

Vera-Villarroel, P., \& Lillo, S. (2006). La investigación actual en psicología clínica en Chile: un análisis a partir de la producción. Terapia Psicológica, 24(2), 221-230.

Vera-Villarroel, P., López-López, W., Lillo, S., \& Silva, L. M. (2011). Scientific production in LatinAmerican psychology: An analysis of research in different countries. Revista Latinoamericana de Psicología, 43(1), 95-104.

Vera-Villarroel, P., \& Mustaca, A. (2006). Investigaciones en psicología clínica basadas en la evidencia en Chile y Argentina. Revista Latinoamericana de Psicología, 38(3), 551-565.

Villegas, J. F. (2012). Autobiografía. En H. Klappenbach \& R. León (Eds.), Historia de la psicología iberoamericana en autobiografías (pp. 275-312). Lima: Universitaria.

Villegas, J. F., \& Rodríguez, M. (2005). Historia de la investigación científica de la psicología en Chile (Vol. 1). Santiago: Universidad Mariano Egaña. 